\title{
Article original / Original article \\ Profil métallique sanguin chez des volontaires sains : 10 ans après
}

\section{Blood metal profile in healthy volunteers: 10 years later}

\author{
Alexandre Cesbron ${ }^{1 \star}$, Elodie Saussereau ${ }^{1}$, Loïc Mahieu ${ }^{1}$, Isabelle Couland ${ }^{2}$, Tatiana Enault ${ }^{2}$, \\ Michel Guerbet ${ }^{3}$, Jean-Pierre Goullé, ${ }^{1,3}$ \\ ${ }^{1}$ Laboratoire de Pharmacocinétique et de Toxicologie Cliniques, Groupe Hospitalier du Havre, BP 24, 76083 Le Havre Cedex, France \\ 2 Service de Médecine Préventive, Groupe Hospitalier du Havre, BP 24, 76083 Le Havre Cedex, France \\ 3 UFR de Médecine et de Pharmacie, 22 boulevard Gambetta, 76183 Rouen Cedex 1, France
}

\begin{abstract}
Résumé - Objectifs : Dix ans après avoir mesuré les concentrations normales d'une trentaine d'éléments métalliques dans le sang et le plasma, par plasma à couplage inductif relié à un détecteur de masse (ICP-MS), il nous a semblé utile d'évaluer l'impact des modifications éventuelles de notre environnement sur ces teneurs. Méthodes : Cent six personnels hospitaliers volontaires des deux sexes, indemnes de toute affection, sans exposition professionnelle aux métaux, non traités par du lithium ou du strontium, ne prenant pas d'oligo-élément ni de complément vitaminique, non porteurs de prothèse métallique, ont été inclus. La consommation de tabac et le port d'amalgame dentaire sont renseignés. Un dosage multiélémentaire de 26 métaux par ICP-MS ( $\mathrm{Li}, \mathrm{Be}, \mathrm{V}, \mathrm{Cr}, \mathrm{Mn}, \mathrm{Co}, \mathrm{Ni}, \mathrm{Cu}, \mathrm{Zn}, \mathrm{As}, \mathrm{Se}, \mathrm{Rb}$, $\mathrm{Sr}, \mathrm{Mo}, \mathrm{Ag}, \mathrm{Cd}, \mathrm{Sn}, \mathrm{Te}, \mathrm{Ba}, \mathrm{W}, \mathrm{Pt}, \mathrm{Hg}, \mathrm{TI}, \mathrm{Pb}, \mathrm{Bi}$ et U) est effectué sur sang total et sur plasma. Résultats : Parmi les résultats notables, nous constatons que la plombémie médiane est divisée par deux, alors que les dosages ont été réalisés dans les mêmes conditions analytiques dans une cohorte similaire à celle analysée en $2003(12,5 \mu \mathrm{g} / \mathrm{L}$ versus $26,3 \mu \mathrm{g} / \mathrm{L}-p<0,0001)$. Une concentration sanguine plus élevée de cadmium est mesurée chez les fumeurs par rapport aux non fumeurs $(1,18 \mu \mathrm{g} / \mathrm{L}$ versus $0,27 \mu \mathrm{g} / \mathrm{L}-p<0,0001)$. Aucun métal et en particulier le mercure sanguin ou plasmatique n'est significativement augmenté chez les porteurs d'amalgames. Conclusion : Au cours des dix dernières années la plombémie médiane a été divisée par deux. S'agissant du métal le plus fréquemment surveillé en biologie clinique et en raison des concentrations mesurées, les dosages de plomb en routine devraient désormais être réalisés par ICP-MS.
\end{abstract}

Mots clés : ICP-MS, métaux, plomb, sang, plasma

\begin{abstract}
Objective: Ten years after determining the normal concentrations in whole blood and plasma of approximately thirty elements by inductively coupled plasma mass spectrometry, it seemed useful to assess the impact of any changes in our environment on these values. Methods: One hundred and six healthy hospital staff volunteers of both sexes, without professional exposure to metals, not treated with lithium or strontium, taking no trace element or vitamin supplements, and without metallic prostheses, were included. Tobacco consumption and the number of dental amalgams were reported. A multi-elementary quantification of 26 metals ( $\mathrm{Li}, \mathrm{Be}, \mathrm{V}, \mathrm{Cr}, \mathrm{Mn}, \mathrm{Co}, \mathrm{Ni}, \mathrm{Cu}, \mathrm{Zn}, \mathrm{As}, \mathrm{Se}, \mathrm{Rb}, \mathrm{Sr}$, Mo, Ag, Cd, Sn, Te, Ba, W, Pt, Hg, TI, Pb, Bi and U) in whole blood and plasma was carried out by ICP-MS. Results: The median blood lead level is halved, while the assays were performed under the same conditions $(12.5 \mu \mathrm{g} / \mathrm{L}$ versus $26.3 \mu \mathrm{g} / \mathrm{L}-p<0.0001)$. The whole blood cadmium concentration is significantly higher in smokers compared with non-smokers $(1.18 \mu \mathrm{g} / \mathrm{L}$ versus $0.27 \mu \mathrm{g} / \mathrm{L}-p<0.0001)$. No metal, and in particular whole blood or plasma mercury, is significantly increased in subjects with amalgams. Conclusion: During this ten-year period, the median blood lead level was halved. Due to the significant blood lead decrease, and being the metal which is the most frequently tested, it is mandatory to perform this analysis by ICP-MS.
\end{abstract}

Key words: ICP-MS, metals, lead, whole blood, plasma

Reçu le 9 février 2013, accepté après modifications le 9 juillet 2013

Publication en ligne le 7 octobre 2013

\footnotetext{
${ }^{\star}$ Correspondance : Alexandre Cesbron, alex.cesbron@ @otmail.fr
} 


\section{Introduction}

En 2003, au cours d'un travail précédent, des valeurs usuelles des métaux et métalloïdes avaient été établies chez 100 sujets volontaires par le dosage multiélémentaire des métaux et métalloïdes dans les milieux biologiques, par plasma à couplage inductif couplé à un détecteur de masse (ICP-MS) $[1,2]$. Ces dernières années, l'exposition à certains éléments est susceptible d'avoir été modifiée. C'est le cas par exemple du plomb, dont la concentration sanguine diminue régulièrement au cours des dernières décennies, en raison notamment de la suppression du plomb dans le carburant ainsi que du renouvellement des canalisations d'eau de consommation [3]. Il nous a donc semblé intéressant d'établir dix ans après de nouvelles valeurs normales, afin de prendre en compte d'éventuelles variations de l'exposition aux éléments métalliques dans une population de volontaires sains.

Nous avons aussi étudié l'impact de différents facteurs sur la concentration en métaux : sexe, tabagisme, présence d'amalgames dentaires. En effet, en ce qui concerne le tabac, sa fumée contient de nombreux composés toxiques, dont des métaux lourds tels le cadmium, le plomb, le mercure, et le chrome, susceptibles de pénétrer dans l'organisme et d'augmenter leur concentration sanguine. Quant aux amalgames dentaires, ils sont constitués d'un mélange de mercure et d'autres métaux en poudre : argent, cuivre, étain, zinc. Le mercure qui entre dans la composition alimente une controverse, notamment sur une éventuelle toxicité du métal pouvant être relargué à partir des amalgames.

La technique de dosage utilisée est la même que celle de la précédente étude. Il s'agit d'un dosage par ICP-MS, technique sensible et spécifique, permettant un dosage rapide et simultané d'une trentaine de métaux $[1,2]$.

\section{Matériels et méthodes}

\subsection{Population d'étude}

106 personnels hospitaliers volontaires des deux sexes, indemnes de toute affection, sans exposition professionnelle aux métaux, non traités par du lithium ou du strontium, ne prenant pas d'oligo-élément ni de complément vitaminique, non porteurs de prothèse métallique, sont inclus. La consommation de tabac et le port d'amalgames dentaires sont renseignés.

\subsection{Prélèvements}

Le sang est prélevé dans des tubes sous vide en PET de $6 \mathrm{~mL}$ pour éléments traces contenant $10 \mathrm{mg}$ d'EDTA diK (vacutainer réf. 368381, Becton Dickinson, Le Pont de Claix, France). Les prélèvements sont effectués par le service de médecine préventive au cours d'un bilan biologique. Des aiguilles standard en inox vacutainer precisionglide ${ }^{\mathrm{TM}}$ (Becton Dickinson) sont utilisées. Afin de limiter le relargage de métaux à partir de l'aiguille, les tubes pour éléments traces sont prélevés en dernier, après ceux du bilan biologique.

\subsection{Instrumentation}

L'ICP-MS utilisé pour le dosage est un plasma à couplage inductif de type ThermoElectron X Series couplée à un spectromètre de masse équipé d'une cellule de collision, modèle X7/CCT (ThermoFischer, Courtabœuf, France). L'appareil comporte une torche en quartz de 1,5 $\mathrm{mm}$, un nébuliseur concentrique en verre borosilicaté de $1 \mathrm{~mL}$ (type Meinhard de Marque Glass Expansion), une chambre simple de nébulisation à bille d'impact en quartz munie d'un refroidisseur à effet Peltier régulant sa température à $3{ }^{\circ} \mathrm{C}$, et un passeur d'échantillons de type CETAC ASX-510. L'ensemble des acquisitions est enregistré à l'aide d'une station informatique dotée du logiciel d'analyse PlasmaLab version 2.5.9 sous Windows NT. Les paramètres instrumentaux sont les suivants : puissance de la torche $1200 \mathrm{~W}$; débits d'argon : plasmagène : $15 \mathrm{~L} / \mathrm{min}$, nébuliseur : 0,80 L/min, auxiliaire : $0,80 \mathrm{~L} / \mathrm{min}$; interface : cônes échantillonneur et écorceur en nickel de diamètres respectifs de $1 \mathrm{~mm}$ et de $0,4 \mathrm{~mm}$; vide au niveau de l'interface : 1,9 mbar et du quadripôle : $1,6 \times 10^{-7}$ mbar. Le gaz et les réactifs sont de qualité certifiée :

- argon à 99,999 \%, Linde gas (Gargenville, France) ;

- acide nitrique suprapur, Triton X 100 Merck (Darmstadt, Allemagne);

- N-butanol rectapur, Prolabo WWR (Fontenay-sous-Bois, France);

- solution de calibration multiélémentaire : Atasol - MixSP 768 B Analytika (Prague, République tchèque);

- étalons internes : rhodium (Rh) $1 \mathrm{~g} / \mathrm{L}$, indium (In) $1 \mathrm{~g} / \mathrm{L}$ C.A.S. (Courtage Analyse Services, Mont-Saint-Aignan, France). Ces solutions mères servent à préparer une solution fille à $10 \mathrm{mg} / \mathrm{L}$ dans l'acide nitrique à $2 \%$ );

- l'eau est purifiée sur Synergy UV, Millipore (Saint-Quentin-en-Yvelines, France).

- diluant : 0,1 mL de chaque solution fille d'étalon interne (In et $\mathrm{Rh}$ à $10 \mathrm{mg} / \mathrm{L}$ ) - 0,1 mL de triton X 100 pour le plasma et $1 \mathrm{~mL}$ pour le sang - $10 \mathrm{~mL}$ d'acide nitrique $5 \mathrm{~mL}$ de n-butanol - eau QSP $1000 \mathrm{~mL}$.

\subsection{Méthode}

Les dosages sont réalisés dans le plasma et le sang total $(0,3 \mathrm{~mL})$ après dilution au dixième en milieu acide $(2,6 \mathrm{~mL}$ de diluant) auxquels on ajoute $0,1 \mathrm{~mL}$ d' acide nitrique à $2 \%$. Pour le plasma, l'étalonnage est effectué en milieu aqueux, alors qu'il est fait appel à la méthode des ajouts dosés pour le sang total conformément à la validation technique antérieure [4]. Les 27 éléments suivants sont quantifiés : Li, Be, V, Cr, Mn, $\mathrm{Co}, \mathrm{Ni}, \mathrm{Cu}, \mathrm{Zn}, \mathrm{As}, \mathrm{Se}, \mathrm{Rb}, \mathrm{Sr}, \mathrm{Mo}, \mathrm{Ag}, \mathrm{Cd}, \mathrm{Sn}, \mathrm{Te}, \mathrm{Ba}, \mathrm{W}$, $\mathrm{Pt}, \mathrm{Hg}, \mathrm{Tl}, \mathrm{Pb}, \mathrm{Bi}$ et $\mathrm{U}$. Pour le vanadium et le chrome, la présence d'une interférence aux masses de mesure 51 et 52 impose l'utilisation d'une cellule de collision réactionnelle, selon une procédure déjà décrite [5]. Pour le mercure plasmatique, en raison d'un «effet mémoire », il est fait appel à une technique spécifique en mode monoélémentaire [6]. L'exactitude des mesures est garantie par l'emploi de plasma et de sang certifiés, Séronorm ${ }^{\circledR}$ (SERO, Billingstade, Norvège). La qualité des résultats obtenus est vérifiée avec chaque série, à l'aide de 
Tableau I. Valeurs usuelles dans le sang total chez des volontaires des deux sexes. Les résultats sont exprimés en $\mu \mathrm{g} / \mathrm{L}$ ou ppb.

\begin{tabular}{|c|c|c|c|c|}
\hline \multirow[b]{2}{*}{ Élément } & \multicolumn{2}{|c|}{2003} & \multicolumn{2}{|r|}{2012} \\
\hline & $\begin{array}{l}\text { Médiane } \\
(n=100)\end{array}$ & $\begin{array}{c}\text { Dispersion } \\
5^{\mathrm{e}}-95^{\mathrm{e}} \text { percentile }\end{array}$ & $\begin{array}{l}\text { Médiane } \\
(n=106)\end{array}$ & $\begin{array}{c}\text { Dispersion } \\
5^{\mathrm{e}-95^{\mathrm{e}} \text { percentile }}\end{array}$ \\
\hline${ }^{7}$ Lithium & NR & NR & $<1,2$ & $<1,4$ \\
\hline${ }^{9}$ Béryllium & $<0,14$ & $<0,14$ & $<1,4$ & $<0,14$ \\
\hline${ }^{51}$ Vanadium* & NR & NR & $<0,21$ & $<0,21-0,35$ \\
\hline${ }^{52}$ Chrome* & NR & NR & 0,55 & $<0,33-0,87$ \\
\hline${ }^{55}$ Manganèse & 7,6 & $5,0-12,8$ & 8,6 & $5,9-13,3$ \\
\hline${ }^{59}$ Cobalt & 0,25 & $<0,06-0,64$ & 0,27 & $0,20-0,63$ \\
\hline${ }^{60} \mathrm{Nickel}$ & 2,14 & $<0,63-4,18$ & 0,80 & $<0,63-1,36$ \\
\hline${ }^{65}$ Cuivre & NR & NR & 937 & $743-1513$ \\
\hline${ }^{66}$ Zinc & NR & NR & 5617 & $4220-7198$ \\
\hline${ }^{75}$ Arsenic & 5,0 & $2,6-17,8$ & 1,87 & $1,08-7,10$ \\
\hline${ }^{82}$ Selenium & 119 & 89-154 & 110 & $85-142$ \\
\hline${ }^{85}$ Rubidium & 1680 & $1289-2358$ & 1794 & $1361-2554$ \\
\hline${ }^{88}$ Strontium & 16 & $9-41$ & 15 & $8-35$ \\
\hline${ }^{95}$ Molybdène & 2,88 & $0,77-7,86$ & NR & NR \\
\hline${ }^{98}$ Molybdène & NR & NR & 0,40 & $0,26-0,77$ \\
\hline${ }^{107}$ Argent & 1,40 & $0,69-4,51$ & $<0,27$ & $<0,27-0,49$ \\
\hline${ }^{111}$ Cadmium & 0,31 & $0,15-2,04$ & 0,34 & $0,13-1,82$ \\
\hline${ }^{118}$ Étain & 1,11 & $0,11-1,75$ & 0,34 & $0,16-0,64$ \\
\hline${ }^{125}$ Tellure & 0,16 & $0,11-0,45$ & 0,14 & $0,08-0,26$ \\
\hline${ }^{137}$ Baryum & 59 & $46-78$ & 0,44 & $0,10-6,87$ \\
\hline${ }^{182}$ Tungstène & $<0,03$ & $<0,03-0,08$ & $<0,03$ & $<0,03$ \\
\hline${ }^{195}$ Platine & $<0,01$ & $<0,01$ & $<0,01$ & $<0,01$ \\
\hline${ }^{202}$ Mercure & 3,0 & $0,94-8,13$ & 1,72 & $0,59-5,12$ \\
\hline${ }^{205}$ Thallium & 0,023 & $0,011-0,035$ & 0,021 & $0,004-0,034$ \\
\hline${ }^{208}$ Plomb & 26 & $11,4-62,8$ & 12,5 & $5,4-39,3$ \\
\hline${ }^{209}$ Bismuth & $<0,007$ & $<0,007$ & $<0,007$ & $<0,007-0,024$ \\
\hline${ }^{238}$ Uranium & 0,004 & 0,006 & $<0,002$ & $0,002-0,003$ \\
\hline
\end{tabular}

* Dosage en cellule de collision réaction; NR : dosage non réalisé.

contrôles internes. Notre laboratoire participe régulièrement à un programme international de comparaisons interlaboratoires sur sang total et sur plasma par ICP-MS organisé par l'Institut national de santé publique du Québec (Canada).

\section{Résultats}

Ils sont exprimés en $\mu \mathrm{g} / \mathrm{L}(=\mathrm{ng} / \mathrm{mL}$ ou $\mathrm{ppb})$ pour le sang total (tableau I) et le plasma (tableau II). Pour chaque élément, la concentration médiane, ainsi que les valeurs du $5^{\mathrm{e}}$ au $95^{\mathrm{e}}$ percentile sont indiquées. Les mesures inférieures à la limite de quantification, selon la règle communément admise, sont considérées égales à la moitié de la limite de quantification. Les tests statistiques utilisés sont des tests $t$ de comparaison des moyennes. Une colonne rappelle les résultats obtenus dans la cohorte prélevée en 2003.

\subsection{Comparaison des résultats obtenus en 2012 avec ceux de 2003}

Les nouvelles valeurs usuelles obtenues avec cette cohorte de 106 volontaires sont comparées à celles mesurées dans la série des 100 sujets inclus en 2003 . Les éléments ${ }^{51} \mathrm{~V},{ }^{52} \mathrm{Cr}$, sont manquants dans cette comparaison, n'ayant pas été quantifiés en 2003 du fait de l'absence de cellule de collision en 2003. Le changement d'isotope pour le lithium et le molybdène ${ }^{6} \mathrm{Li}$ et ${ }^{95} \mathrm{Mo}$ en 2003 , puis ${ }^{7} \mathrm{Li}$ et ${ }^{98} \mathrm{Mo}$ en 2012 n'autorise pas les comparaisons. Enfin, la présence de baryum, liée à l'emploi de tubes en verre en 2003, nous a contraints à également exclure cet élément.

Dans le sang total, nous constatons des variations significatives pour plusieurs éléments (figure 1). Ainsi, la plombémie médiane a été divisée par deux $(12,5 \mu \mathrm{g} / \mathrm{L}$ vs $26,3 \mu \mathrm{g} / \mathrm{L}$, $p<0,0001)$. On observe également une diminution significative des concentrations médianes de l'arsenic $(1,9 \mu \mathrm{g} / \mathrm{L}$ 
Tableau II. Valeurs usuelles dans le plasma chez des sujets volontaires des deux sexes. Les résultats sont exprimés en $\mu \mathrm{g} / \mathrm{L}$ ou ppb.

\begin{tabular}{|c|c|c|c|c|}
\hline \multirow[b]{2}{*}{ Élément } & \multicolumn{2}{|r|}{2003} & \multicolumn{2}{|r|}{2012} \\
\hline & $\begin{array}{l}\text { Médiane } \\
(n=100)\end{array}$ & $\begin{array}{c}\text { Dispersion } \\
5^{\mathrm{e}-95^{\mathrm{e}} \text { percentile }}\end{array}$ & $\begin{array}{l}\text { Médiane } \\
(n=100)\end{array}$ & $\begin{array}{c}\text { Dispersion } \\
5^{\mathrm{e}}-95^{\mathrm{e}} \text { percentile }\end{array}$ \\
\hline${ }^{7}$ Lithium & 3,4 & $1,8-18,8$ & 0,64 & $<0,60-1,88$ \\
\hline${ }^{9}$ Béryllium & $<0,10$ & $<0,10$ & $<0,10$ & $<0,10$ \\
\hline${ }^{51}$ Vanadium* & NR & NR & 0,23 & $<0,21-0,38$ \\
\hline${ }^{52}$ Chrome* & NR & NR & $<0,29$ & $<0,29-0,79$ \\
\hline${ }^{55}$ Manganèse & 1,12 & $0,63-2,26$ & 0,65 & $0,35-1,08$ \\
\hline${ }^{59}$ Cobalt & 0,49 & $0,30-1,02$ & 0,30 & $<0,28-0,59$ \\
\hline${ }^{60} \mathrm{Nickel}$ & 2,20 & $<0,28-5,31$ & 0,84 & $0,30-1,26$ \\
\hline${ }^{65}$ Cuivre & 1075 & $794-2023$ & 952 & $627-1659$ \\
\hline${ }^{66}$ Zinc & 726 & $551-925$ & 660 & $510-809$ \\
\hline${ }^{75}$ Arsenic & 6,2 & $4,36-14,2$ & 2,19 & $1,55-7,58$ \\
\hline${ }^{82}$ Selenium & 112 & 79-141 & 92 & 73-110 \\
\hline${ }^{85}$ Rubidium & 148 & $101-358$ & 308 & $207-1178$ \\
\hline${ }^{88}$ Strontium & 29 & $18-75$ & 27 & $17-67$ \\
\hline${ }^{95}$ Molybdène & 0,96 & $0,67-1,68$ & NR & NR \\
\hline${ }^{98}$ Molybdène & NR & NR & 0,61 & $0,36-1,15$ \\
\hline${ }^{107}$ Argent & NR & NR & $<0,57$ & $<0,57-0,80$ \\
\hline${ }^{111}$ Cadmium & 0,03 & $<0,03-0,05$ & $<0,03$ & $<0,03-0,04$ \\
\hline${ }^{118}$ Étain & 1,8 & $<1,0-2,7$ & $<1,0$ & $<1,0$ \\
\hline${ }^{125}$ Tellure & 0,06 & $<0,04-0,13$ & 0,05 & $<0,04-0,09$ \\
\hline${ }^{137}$ Baryum & 111 & $90-154$ & 0,52 & $0,22-1,22$ \\
\hline${ }^{182}$ Tungstène & $<0,62$ & $<0,62-0,75$ & $<0,62$ & $<0,62$ \\
\hline${ }^{195}$ Platine & 0,38 & $<0,02-0,92$ & $<0,02$ & $<0,02-0,04$ \\
\hline${ }^{202}$ Mercure & NR & NR & 0,36 & $0,09-1,01$ \\
\hline${ }^{205}$ Thallium & 0,06 & $0,01-0,24$ & 0,04 & $0,03-0,06$ \\
\hline${ }^{209}$ Bismuth & $<0,01$ & $<0,01-0,40$ & $<0,01$ & $<0,01-0,04$ \\
\hline${ }^{238}$ Uranium & 0,007 & $0,004-0,011$ & 0,003 & $<0,002-0,006$ \\
\hline
\end{tabular}

${ }^{*}$ Dosage en cellule de collision en réaction.

vs $5,0 \mu \mathrm{g} / \mathrm{L}, p<0,001)$, de l'étain $(0,34 \mu \mathrm{g} / \mathrm{L} v s 1,11 \mu \mathrm{g} / \mathrm{L}$, $p<0,001)$, du mercure $(1,7 \mu \mathrm{g} / \mathrm{L} v s 3,0 \mu \mathrm{g} / \mathrm{L}, p<0,0001)$, et du nickel $(0,80 \mu \mathrm{g} / \mathrm{L} v s 2,14 \mu \mathrm{g} / \mathrm{L}, p<0,0001)$. La concentration médiane de manganèse $(8,6 \mu \mathrm{g} / \mathrm{L} v s 7,6 \mu \mathrm{g} / \mathrm{L}, p=0,001)$ est supérieure à celle obtenue en 2003. Il n'y a pas de différence pour tous les autres éléments.

Dans le plasma, des variations significatives sont constatées pour neuf éléments (figure 2). Nous remarquons une diminution pour le nickel $(0,84 \mu \mathrm{g} / \mathrm{L} v s 2,14 \mu \mathrm{g} / \mathrm{L}, p<0,0001)$, l'étain $(0,50 \mu \mathrm{g} / \mathrm{L} v s 1,11 \mu \mathrm{g} / \mathrm{L}, p<0,0001)$, le zinc $(660 \mu \mathrm{g} / \mathrm{L}$ vs $726 \mu \mathrm{g} / \mathrm{L}, p<0,0001)$, l'arsenic $(2,2 \mu \mathrm{g} / \mathrm{L}$ vs $6,2 \mu \mathrm{g} / \mathrm{L}$, $p<0,0001)$, le cobalt $(0,30 \mu \mathrm{g} / \mathrm{L}$ vs $0,49 \mu \mathrm{g} / \mathrm{L}, p<0,0001)$, le cuivre $(952 \mu \mathrm{g} / \mathrm{L} v s 1075 \mu \mathrm{g} / \mathrm{L}, p<0,01)$, le manganèse $(0,65 \mu \mathrm{g} / \mathrm{L} v s 1,12 \mu \mathrm{g} / \mathrm{L}, p<0,001)$ et le sélénium $(92 \mu \mathrm{g} / \mathrm{L} v s$ $112 \mu \mathrm{g} / \mathrm{L}, p<0,0001)$. À l'inverse, le rubidium plasmatique $(303 \mu \mathrm{g} / \mathrm{L}$ vs $148 \mu \mathrm{g} / \mathrm{L}, p<0,001)$ est augmenté.

\subsection{Influence du sexe}

Le profil métallique des hommes $(n=29)$ est comparé à celui des femmes $(n=77)$. Des différences significatives, dépendantes du sexe sont constatées (figure 3) : chez les hommes, la plombémie médiane est supérieure à celle des femmes
$(19,8 \mu \mathrm{g} / \mathrm{L} v s$ 11,1 $\mu \mathrm{g} / \mathrm{L}, p<0,001)$. La concentration médiane de zinc plasmatique chez l'homme est supérieure à celle de la femme $(718 \mu \mathrm{g} / \mathrm{L} v s 647 \mu \mathrm{g} / \mathrm{L}, p<0,01)$; alors que pour le cuivre, elle est supérieure chez la femme $(965 \mu \mathrm{g} / \mathrm{L} v s$ $817 \mu \mathrm{g} / \mathrm{L}, p<0,001)$.

\subsection{Influence du tabagisme}

La concentration médiane de cadmium dans le sang total est plus élevée chez les fumeurs $(n=29)$ que chez les non fumeurs $(n=77)(1,18 \mu \mathrm{g} / \mathrm{L} v s 0,27 \mu \mathrm{g} / \mathrm{L}, p<0,0001)$ (figure 5). La plombémie médiane est également supérieure chez les fumeurs $(18,7 \mu \mathrm{g} / \mathrm{L}$ vs $11,8 \mu \mathrm{g} / \mathrm{L}, p<0,05)$.

\subsection{Influence du port d'amalgames dentaires}

Des variations du profil métallique dans notre cohorte, dues à la présence d'amalgames dentaires sont recherchées, entre porteurs $(n=83)$ et non porteurs d'amalgames $(n=23)$. Aucune différence significative n'est mise en évidence entre les deux groupes, en particulier pour le mercure plasmatique 

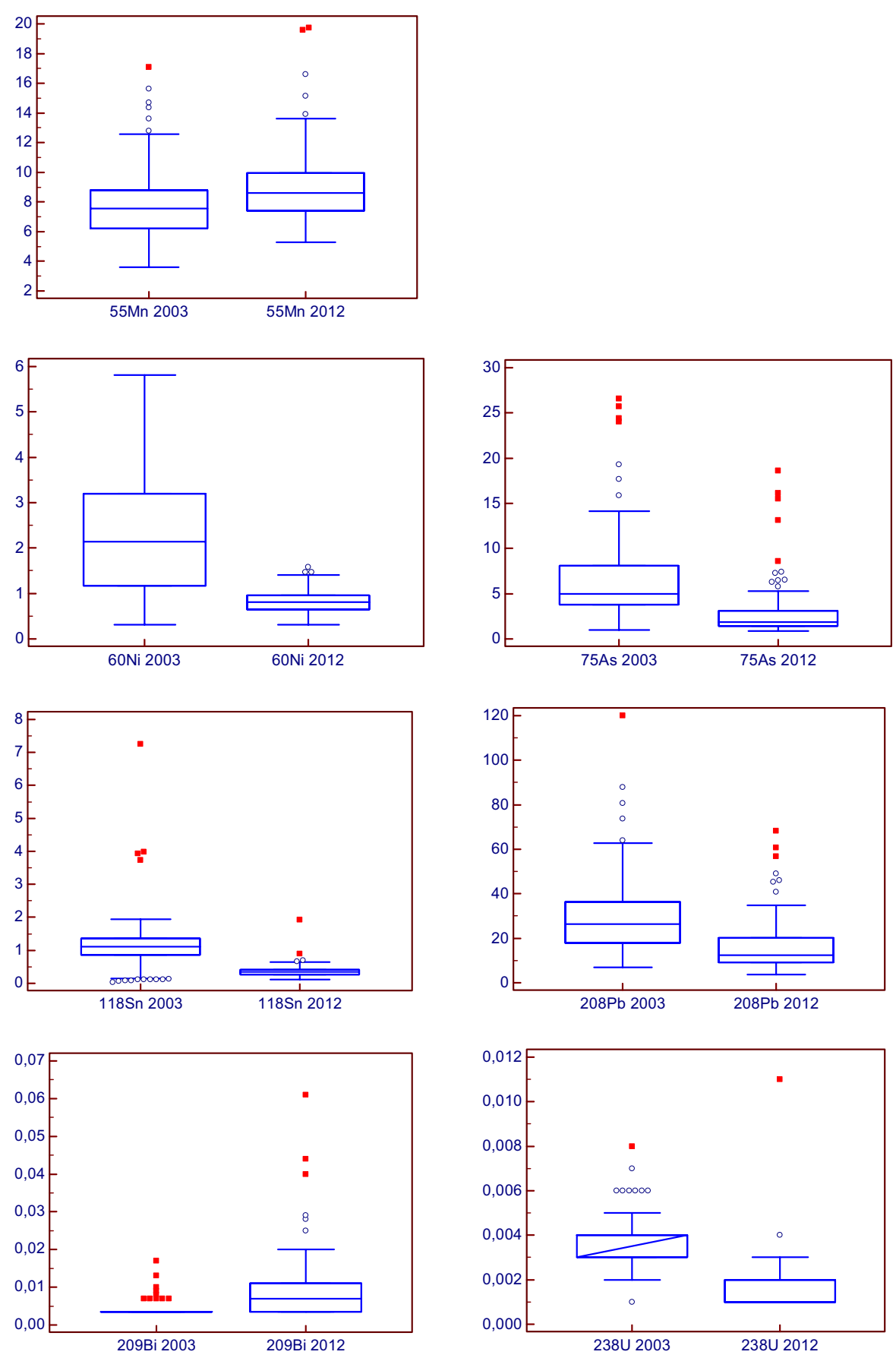

Fig. 1. Comparaison des valeurs usuelles 2003 et 2012 pour certains métaux dans le sang total.

considéré comme le meilleur marqueur de la présence de mercure inorganique $(0,38 \mu \mathrm{g} / \mathrm{L}$ vs $0,28 \mu \mathrm{g} / \mathrm{L}, p=0,11)$ (figure 6). Il n'est pas constaté de lien entre le nombre d'amalgames et la concentration en mercure $(r=0,23, p=0,03)$.

\section{Discussion}

Cette étude montre plusieurs différences significatives par rapport à celle réalisée près de dix ans auparavant.
Depuis 2003, nous constatons en particulier une diminution très importante de la plombémie médiane puisque celle-ci est divisée par deux. En 2005, Testud rapportait déjà que la plombémie médiane avait chuté de $125 \mu \mathrm{g} / \mathrm{L}$ à $65 \mu \mathrm{g} / \mathrm{L}$ en dix ans [3]. Cette baisse considérable peut s'expliquer par la disparition progressive du carburant au plomb, puisqu'elle est totale depuis 2000. En effet, jusqu'au début des années quatre-vingt, les émissions des véhicules à moteur essence étaient responsables de $90 \%$ du plomb présent dans l'atmosphère des grandes villes, soit des taux pouvant aller jusqu'à 

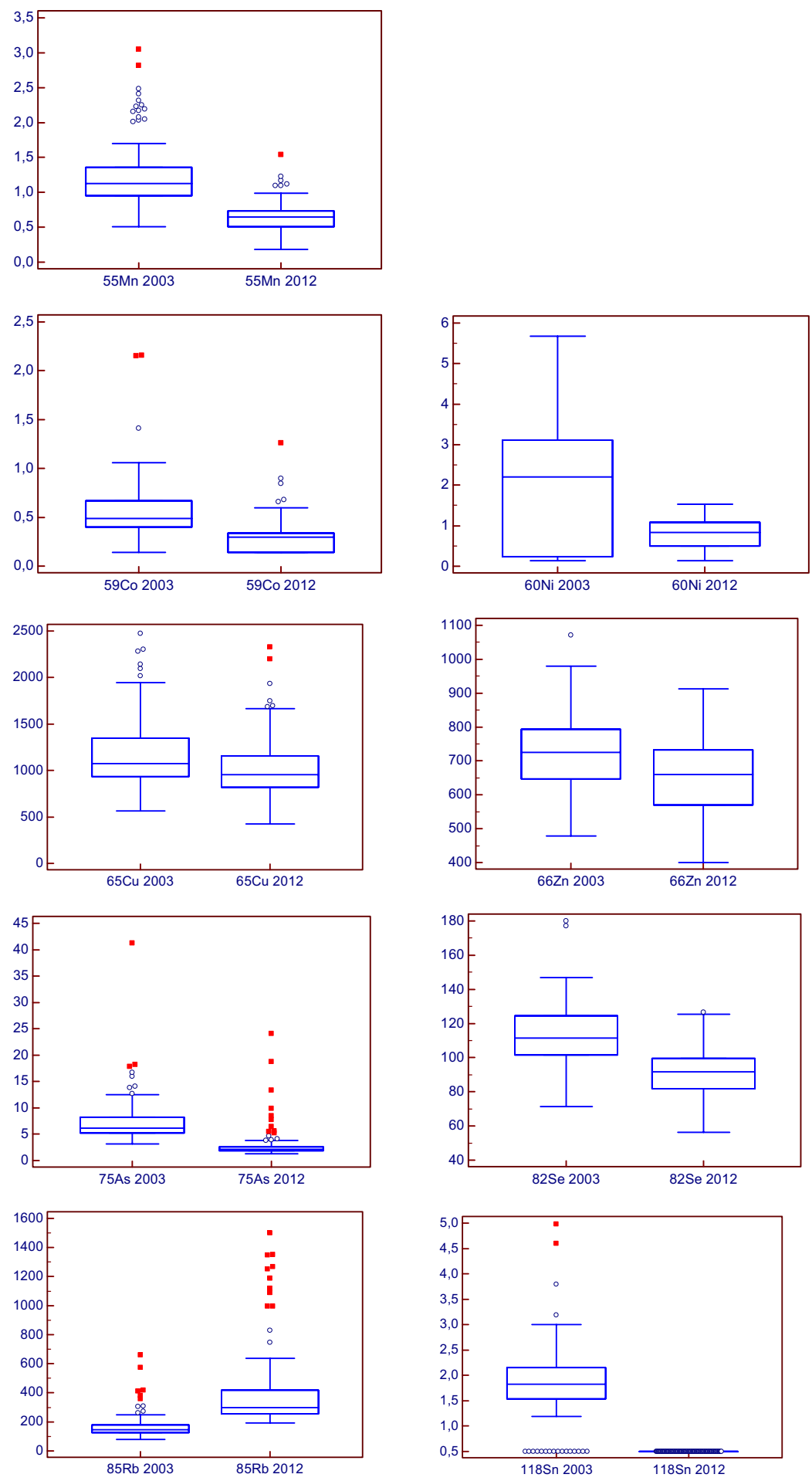

Fig. 2. Comparaison des valeurs usuelles 2003 et 2012 pour certains métaux dans le plasma.

$5 \mu \mathrm{g} / \mathrm{m}^{3}$. Depuis 2002, la réglementation impose une valeur limite à $0,5 \mu \mathrm{g} / \mathrm{m}^{3}$ [3]. La diminution progressive de la plombémie médiane peut éventuellement s'expliquer pour une grande part en raison du stockage osseux du plomb, dont la demi-vie est de 10 à 30 ans, responsable d'une élimination très lente du métal de l'organisme [7]. Une diminution des teneurs alimentaire et hydrique doit être évoquée, pouvant contribuer à réduire les apports d'un métal dont la capacité rénale d'élimination est faible. Concernant le remplacement progressif des canalisations en plomb dans les grandes villes, qui représente une réelle source d'exposition par la consommation d'eau pouvant être contaminée, ainsi que la présence de peintures à base de céruse dans des logements anciens, de telles hypothèses ne peuvent être retenues dans cette cohorte. En effet, 


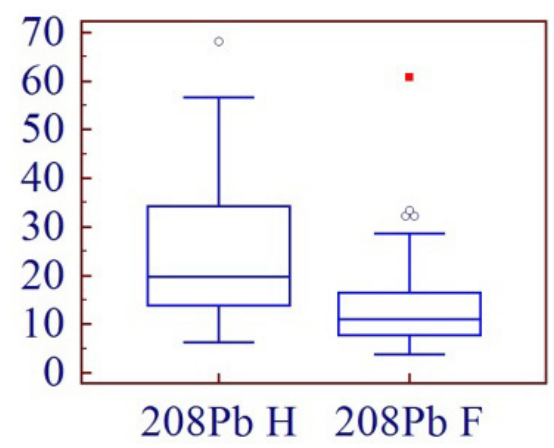

Fig. 3. Comparaison des concentrations en plomb entre les hommes $(\mathrm{H})$ et les femmes $(\mathrm{F})$ dans le sang total.

la ville du Havre a été détruite à $90 \%$ lors des bombardements de 1944, aussi l'essentiel des logements a été construit bien après cette date. Les nombreuses mesures de plombémie inférieures à $10 \mu \mathrm{g} / \mathrm{L}$ posent la question de la technique de dosage du plomb. En effet, l'immense majorité des laboratoires effectue le dosage du plomb par spectrophotométrie d'absorption atomique. Or, la plombémie médiane est voisine de la limite de quantification de cette technique, soit $10 \mu \mathrm{g} / \mathrm{L}$ [8]. Il apparaît donc désormais indispensable de réaliser les mesures de plombémie par ICP-MS, technique dotée d'une sensibilité cent fois supérieure ( $\mathrm{LQ}<0,07 \mu \mathrm{g} / \mathrm{L})$, comme cela a été recommandé dès 2006, à la suite d'une étude multicentrique [9].

D'autres variations sont constatées pour certains éléments par rapport à 2003. Elles peuvent être expliquées en partie par le choix d'un nouveau tube de prélèvement en PET pour élément traces à la place d'un tube de verre, mais également d'un anticoagulant différent, l'EDTA à la place de l'héparinate de sodium. C'est le cas de l'étain et de l'arsenic, dans le plasma et le sang total, ainsi que du cobalt et du selenium plasmatiques qui sont significativement diminués en 2012 comparativement à 2003, et du rubidium qui est augmenté. Ces variations avaient été mises en évidence au cours d'une précédente étude menée par notre équipe, démontrant l'influence du matériau utilisé pour le tube et de l'anticoagulant utilisés sur la teneur en éléments trace du matériel de recueil [10]. Les baisses des concentrations du nickel dans le plasma et le sang total, et du manganèse plasmatique peuvent être liées au protocole de prélèvement, effectué au moyen de seringues avec aiguille en acier inoxydable, constitués d'un alliage de nickel-fer-chrome, et contenant ces trois métaux [11]. Le prélèvement est effectué au cours d'un bilan biologique de médecine préventive, le tube en PET pour le dosage des métaux étant prélevé en dernier, l'aiguille est donc rincée par le sang destiné à remplir les autres tubes du bilan. Pour s'affranchir totalement de ce problème, la solution idéale serait l'utilisation d'aiguille en téflon sans aucune trace de métal. Nous constatons une baisse du cuivre, du zinc, oligo-éléments essentiels apportés par l'alimentation, pouvant être expliquée par la variabilité des apports. Il en est de même pour le mercure du sang total, qui est le reflet du méthylmercure alimentaire, provenant principalement de produits de la mer.

Nous observons aussi une concentration plasmatique de zinc plus élevée chez l'homme, et de cuivre plus élevée chez la femme. Ces différences sont connues pour les deux éléments.
Les femmes ont une zincémie légèrement inférieure d'environ $1 \mu \mathrm{mol} / \mathrm{L}$, soit $65 \mu \mathrm{g} / \mathrm{L}$ [12]. Dès 1982, Helgeland et coll. mettaient en évidence un cuivre plasmatique plus élevé chez les femmes que chez les hommes $(18,4 \mu \mathrm{mol} / \mathrm{L}$ soit $1168 \mu \mathrm{g} / \mathrm{L} v s$ $16,5 \mu \mathrm{mol} / \mathrm{L}$ soit $1048 \mu \mathrm{g} / \mathrm{L}$ ) [13]. Par ailleurs, chez la femme, la grossesse est une période au cours de laquelle la zincémie médiane diminue et la cuprémie médiane augmente [14]. Une limite de notre cohorte réside dans une répartition inhomogène entre les hommes $(n=29)$ et les femmes $(n=77)$, contrairement à celle de 2003 où la parité avait été parfaitement respectée. Le nombre plus élevé de femmes pourrait expliquer la baisse de la zincémie observée en dix ans, mais ceci est en contradiction avec l'évolution de la cuprémie qui diminue elle aussi. La plombémie supérieure chez l'homme est une notion connue, puisque nous avions établi des normales inférieures à $70 \mu \mathrm{g} / \mathrm{L}$ chez l'homme, inférieures à $60 \mu \mathrm{g} / \mathrm{L}$ chez la femme $[1,2]$.

L'étude de l'influence du tabagisme montre une cadmiémie significativement plus élevée chez les sujets fumeurs, conformément aux données de la littérature. Ainsi, Testud rapporte une cadmiémie normale inférieure à $1,5 \mu \mathrm{g} / \mathrm{L}$ chez les non fumeurs, inférieure à $5 \mu \mathrm{g} / \mathrm{L}$ chez les fumeurs [3]. Le plomb sanguin est également augmenté avec le tabagisme, ce qui est en accord avec les résultats d'une étude sur l'exposition au plomb de la population canadienne, rassemblant 5319 sujets [15]. Il n'a par contre pas été retrouvé d'augmentation significative d'autres métaux contenus dans la fumée de cigarettes, tels que le chrome ou le mercure.

Enfin, la controverse sur une toxicité éventuelle du mercure liée au port d'amalgames dentaires nous a conduits à évaluer son influence sur la concentration plasmatique du mercure. Bergdahl et coll. rapportent que la concentration en mercure plasmatique augmente en moyenne de $0,2 \mu \mathrm{g} / \mathrm{L}$ pour dix surfaces d'amalgame [16]. Notre travail montre que les porteurs d'amalgames ont une concentration médiane du mercure plasmatique sensiblement plus élevée que les non porteurs d'amalgame $(0,38 \mu \mathrm{g} / \mathrm{L}$ vs $0,28 \mu \mathrm{g} / \mathrm{L})$, mais cette différence sur un échantillon réduit n'est pas significative $(p=0,11)$. Par ailleurs, il n'est observé aucune corrélation entre le nombre d'amalgames portés par les sujets volontaires et le mercure plasmatique. De plus, aucun des autres métaux entrant dans la composition chimique des amalgames (argent, cuivre, étain, zinc) n'est augmenté chez nos 83 volontaires qui en sont porteurs. Les résultats de cette étude abondent dans le sens du rapport de l'Afssaps rédigé en octobre 2005, qui confirmait l'absence d'augmentation au-delà des normes du mercure plasmatique ou du mercure sanguin total dans toutes les études publiées sur le sujet [17]. Ce document confirmait le rapport bénéfice-risque favorable de l'amalgame : absence de toxicité prouvée, peu coûteux, facile à mettre en œuvre, meilleure longévité buccale parmi tous les matériaux d'obturation. De plus, l'examen des dossiers médicaux des personnes s'estimant victimes de troubles liés aux amalgames, par un collège de spécialistes, n'a pas permis d'établir un lien entre les divers troubles allégués et la présence du métal : absence de signe clinique caractéristique, dosages de mercure normaux. À ce jour, aucune étude validée n'a pu mettre en évidence un impact quelconque du mercure des amalgames dentaires sur une symptomatologie 

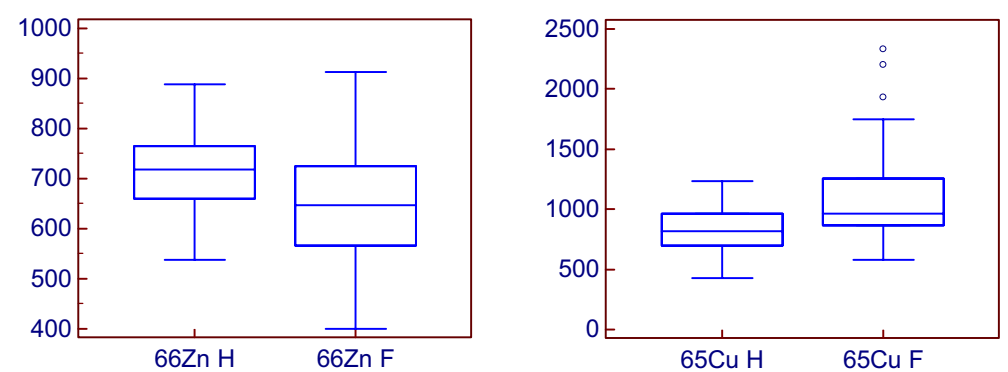

Fig. 4. Comparaison des concentrations en zinc et cuivre entre les hommes $(\mathrm{H})$ et les femmes $(\mathrm{F})$ dans le plasma.

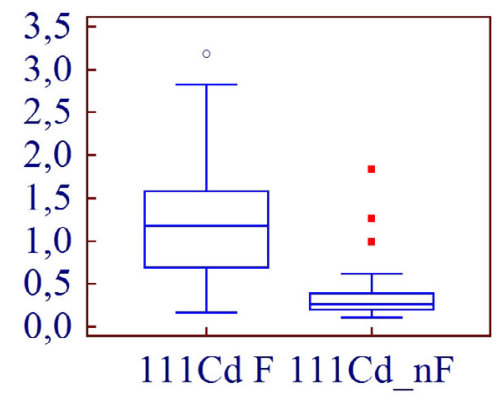

Fig. 5. Comparaison des concentrations en cadmium chez les fumeurs $(\mathrm{F})$ et les non fumeurs $(\mathrm{nF})$ dans le sang total.

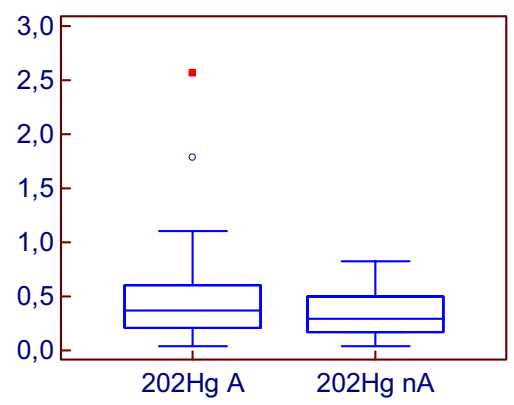

Fig. 6. Comparaison des concentrations en mercure plasmatique entre les porteurs d'amalgames (A) et les non porteurs (nA).

systémique, chez l'adulte ou chez l'enfant. Leur innocuité a d'ailleurs été validée par la commission européenne [18].

\section{Conclusion}

Cette étude montre notamment une baisse très importante de la plombémie médiane, puisque celle-ci est divisée par deux depuis 2003, que cette constatation s'inscrit dans le sens d'une diminution continue sur une période de plus de vingt ans. S'agissant du métal le plus couramment dosé en toxicologie professionnelle, et pour lequel la concentration médiane se rapproche des limites de quantification de la spectrophotométrie d'absorption atomique, il est aujourd'hui indispensable de quantifier le plomb dans le sang par ICP-MS, conformément aux recommandations formulées par la Société Française de Toxicologie Analytique dès 2007. L'ICP-MS est une technique sensible, spécifique, rapide, permettant de doser simultanément une trentaine de métaux. De plus, ce travail souligne une nouvelle fois l'importance du choix et de la nature du matériel de prélèvement : tube, anticoagulant, aiguille, pour le dosage d'un élément métallique. Enfin, aucun lien n'est montré entre la concentration en mercure plasmatique et la présence d'amalgames dentaires, étayant le rapport de l'AFSSAPS de 2005, ainsi que celui de l'Europe qui concluent à l'innocuité du mercure associé au port d'amalgames dentaires.

Conflits d'intérêts. Les auteurs déclarent ne pas avoir de conflits d'intérêts.

\section{Références}

1. Goullé JP, Mahieu L, Neveu N, Bouige D, Casternant J, Lainé G, Nouveau MP, Gehanne R, Lacroix C. Dosage multiélémentaire des métaux et métalloïdes dans les milieux biologiques par ICP-MS : valeurs usuelles chez 100 témoins. Ann Toxicol Anal. 2004; 16(4): 261-268.

2. Goullé JP, Mahieu L, Castermant J, Neveu N, Bonneau L, Lainé G, Bouige D, Lacroix C. Metal and metalloid multi-elementary ICP-MS validation in whole blood, plasma, urine and hair. Reference values. Forensic Sci Int. 2005; 153(1): 39-44.

3. Testud F. Pathologie toxique professionnelle \& environnementale. Éditions Eska, 2005.

4. Goullé JP, Mahieu L, Saussereau E, Bouige D, Groenwont S, Lacroix C. Validation d'une technique de dosage multiélémentaire des métaux et métalloides dans les ongles par ICP-MS. Valeurs usuelles chez 130 sujets volontaires. Ann Toxicol Anal. 2007; 19: 125-134.

5. Goullé JP, Saussereau E, Mahieu L, Coulant I, Plougonven S, Guerbet M, Lacroix C. Validation du dosage du chrome par ICP-MS avec cellule de collision dans les matrices biologiques et concentrations usuelles. Ann Toxicol Anal. 2011; 23(4): 211-216.

6. Goullé JP, Mahieu L, Saussereau E, Lacroix C. Dosage du mercure plasmatique par ICP-MS. Concentrations usuelles chez 53 sujets volontaires. Ann Toxicol Anal. 2008; 20(1): 53-54.

7. Rabinowitz MB. Toxicokinetics of bone lead. Environmental Health Perspectives. 1991; 91: 33-37.

8. Olichon D, Labat L, Poupon J, Bost M, Haufroid V, Moesch C, Nicolas A, Furet Y, Goullé JP, Guillard O, Le Bouil A, Pineau A. Approche analytique de la limite de quantification pour le dosage du plomb sanguin : étude multicentrique. Ann Toxicol Anal. 2007; 19(1): 31-36.

9. Labat L, Olichon D, Poupon J, Bost M, Haufroid V, Moesch C, Nicolas A, Furet Y, Goullé JP, Guillard O, Le Bouil A, Pineau A. Variabilité de la mesure de la plombémie pour de faibles concentrations proches du seuil de $100 \mu \mathrm{g} / \mathrm{L}$ : étude multicentrique. Ann Toxicol Anal. 2006; 18(4): 297-304. 
10. Goullé JP, Mahieu L, Saussereau E, Bouige D, Guerbet M, Lacroix C. Dosage multiélémentaire dans le plasma et le sang total par ICP-MS : influence du matériel de prélèvement. Ann Toxicol Anal. 2008; 20(2): 97-102.

11. Document consulté sur le site http://www.euro-inox.org/ le 30 octobre 2012 .

12. Arnaud J, Favier A. Le zinc. In : Chappuis P (ed.), Les oligoéléments en médecine et biologie. Seconde partie, chapitre 2. Éditions Médicales Internationales, Cachan, France. 1991; 347-397.

13. Helgeland K, Haider T, Jonsen J. Copper and zinc in human serum in Norway relation to geography, sex and age. Scand J Clin Lab Invest. 1982; 42: 35-39.

14. Malek-Mellouli M, Ben Amara F, Loussaief-Ben Amara W, Reziga H. Ann Biol Clin. 2012; 70(4): 423-429.
15. Bushnik T, Haines D, Levallois P, Levesque J, Van Oostdam J, Viau C. Lead and bisphenol A concentrations in the Canadian population. Health Rep. 2010; 21(3): 7-18.

16. Bergdahl IA, Schütz A, Ahlqwist M, Bengtsson C, Lapidus L, Lissner L, Hulten B. Methylmercury and inorganic mercury in serum-correlation to fish consumption and dental amalgam in a cohort of women born in 1922. Environ Res. 1998; 77(1): $20-24$.

17. Le mercure des amalgames dentaires. Rapport, Agence Française de Sécurité Sanitaire des Produits de Santé, octobre 2005, $163 \mathrm{p}$.

18. The safety of dental amalgam and alternative dental restoration materials for patients and users. European Commission, Health \& Consumer Protection Directorate-General, Scientific Committee on Emerging and Newly Identified Health Risks, 6 May 2008, 74 p. 